\title{
Proceso de atención de enfermería en pacientes deprimidos
}

\author{
Zaide Hernández Becerril,* Ma. Guadalupe Nava Galán**
}

\begin{abstract}
RESUMEN
El objetivo de este artículo es dar a conocer un proceso de atención a pacientes con depresión, aplicando el modelo de atención de Dorothea E. Orem, el cual aborda las medidas preventivas más importantes que debe conocer el paciente y la familia, como son: dieta, ejercicio y conocimiento del padecimiento. El proceso se presenta en forma de cuadros para facilitar su manejo y comprensión. Asimismo, pretende dejar la inquietud sobre lo conveniente de emplear modelos de atención de enfermería en problemas de salud que requieren una atención continua por parte del equipo de salud y un conocimiento y compromiso hacia el autocuidado por parte del paciente.
\end{abstract}

Palabras clave: Depresión, proceso enfermero, autocuidado.

\section{Nursing care process in depressed patients}

\begin{abstract}
The aim is to provide a process of care for patients with depression using the care model Dorothea E. Orem, this addresses the most important preventive measures that must know the patient and family, such as diet, exercise, knowledge of the condition. The process is presented in tabular form for ease of handling and understanding, is intended to make the concern about how convenient to use models of nursing care in health problems that require ongoing attention by the health team and an understanding and commitment to self-care by the patient.
\end{abstract}

Key words: Depression, nursing process, self-care.

\section{INTRODUCCIÓN}

$\mathrm{L}$ os antecedentes de la depresión se remontan a algunos escritos médicos de la antigüedad; en el griego clásico y en la época de Hipócrates era conocida como melancolía; en 1725, Sir Richard Blackmore nombró el término que hoy todos conocemos. ${ }^{1}$ $\mathrm{Su}$ incidencia es tan relevante, que hasta la Organización Mundial de la Salud (OMS) estima que para el año 2020, la depresión ocupará la segunda causa de discapacidad a nivel mundial. Dentro de los problemas de salud mental, es uno de los trastornos que puede ser fácilmente diagnosticado en el primer nivel de atención; la OMS estima que entre el 60 y $80 \%$ de las personas con trastorno depresivo pueden ser tratadas por un tipo de terapia breve y el uso de antidepresivos; sin embargo, en algunos países, en el 25\% aproximadamente, sólo el $10 \%$ recibe tales tratamientos. ${ }^{2}$

En México, según datos de la Encuesta Nacional de Epidemiología Psiquiátrica (ENEP), se menciona que seis de

* Licenciada en Enfermería y Obstetricia.
** Maestra en Educación en Enfermería.

Correspondencia: Zaide Hernández Becerril. Insurgentes Sur, Núm. 3877, Col. La Fama, 14269, Delegación Tlalpan, México, D.F. E-mail: rev.enf.neurol@gmail.com; zai_hb@hotmail.com

Este artículo puede ser consultado en versión completa en http://www.medigraphic.com/enfermerianeurologica 
cada 20 mexicanos presentaron algún trastorno mental alguna vez en su vida y tres de cada 20 lo presentaron durante los últimos 12 meses. La depresión es una enfermedad frecuente; de acuerdo con esta encuesta, el $12 \%$ son mujeres y el $7 \%$ hombres quienes han presentado algún trastorno depresivo en algún momento de su vida. ${ }^{3}$ Afecta al $10 \%$, y cada año se atienden a 10,500 personas que han intentado suicidarse. ${ }^{4}$

Para abordar el tema de la depresión se recurrió a la teoría de Dorothea E. Orem aplicada a un proceso de atención de enfermería para implementar medidas y acciones individualizadas. Existen medidas básicas que deben atenderse en estos casos, y es justamente en este proceso que se mencionan. Esto significa un reto para la enfermería, ya que actualmente deben planearse estrategias de atención para lograr la independencia del paciente, y sobre todo fomentar el autocuidado.

Es importante que la enfermería, junto al equipo de salud, se involucren en programas y guíen al paciente sobre la importancia que tiene la depresión. Para la aplicación del proceso de atención enfermero es primordial la aceptación del seguimiento por parte del paciente, ya que implica un compromiso voluntario del que dependerá el éxito para lograr su independencia y autocuidado.

\section{TEORÍA GENERAL DE AUTOCUIDADO}

Dorothea Orem presentó la "Teoría de enfermería del déficit de autocuidado" como una teoría general compuesta por tres teorías relacionadas: ${ }^{5}$ la del autocuidado, que describe el porqué y el cómo las personas cuidan de sí mismas; la del déficit de autocuidado, que describe y explica cómo la enfermería puede ayudar a la gente, y la de sistemas de enfermería, que describe y explica las relaciones que hay que mantener. Para aplicar el modelo de Orem es necesario conocer cómo éste define los conceptos paradigmáticos de persona, enfermería, entorno y salud.
De acuerdo con Dorothe Orem, El autocuidado es la capacidad que tiene una persona para realizar todas las actividades necesarias para vivir y sobrevivir. Orem contempla el concepto "auto" como la totalidad; incluye no sólo las necesidades físicas, sino también las psicológicas y espirituales; el concepto "cuidado" es la totalidad de actividades que una persona inicia para mantener la vida y poder desarrollarse de una forma normal. Orem define el autocuidado como: "La práctica de actividades que los individuos inician y realizan en su beneficio para el mantenimiento de la vida, la salud y el bienestar."

\section{PROCESO DE ATENCIÓN DE ENFERMERÍA}

El proceso de atención de enfermería es un método sistemático que brinda cuidados humanistas eficientes centrados en el logro de resultados esperados, apoyándose en un modelo científico realizado por un profesional de enfermería. Originalmente fue una forma adaptada de resolución de problemas y se clasifica como una teoría deductiva en sí misma. Es un método sistemático y organizado para administrar cuidados individualizados, de acuerdo con el enfoque básico de que cada persona o grupo de personas responde de forma distinta ante una alteración real o potencial de la salud. ${ }^{6}$

Para la elaboración del plan de atención de enfermería fue importante tomar en cuenta la valoración del paciente; por lo tanto, se dio inicio con el análisis del déficit de autocuidado y el balance de autocuidado, de donde surgieron posteriormente los diagnósticos de enfermería. Una vez elaborados los diagnósticos de enfermería, se procedió a la etapa de planificación. En ésta, se plantearon los objetivos tanto para la enfermera como para el paciente, a corto, mediano y largo plazo, de acuerdo a la priorización. Ya planteados los objetivos, se pasó a la implementación de las intervenciones de enfermería de acuerdo al sistema acorde con las necesidades del paciente.

Cuadro I. Análisis del déficit de autocuidado.

\begin{tabular}{|c|c|c|}
\hline \multicolumn{3}{|l|}{ Proceso enfermero en el paciente con depresión } \\
\hline Requisitos & Indicadores del déficit de autocuidado & Sistemas: AE, PC, TC \\
\hline Requisitos universales: & Incumplimiento de la dieta permitida & $\mathrm{X}$ \\
\hline Mantenimiento del aporte suficiente de alimento & Falta de interés por mantener el peso ideal & $\mathrm{X}$ \\
\hline Mantenimiento de balance entre la actividad y el reposo & Consumo excesivo de sal & $\mathrm{X}$ \\
\hline \multirow[t]{4}{*}{ Requisitos de desarrollo y de desviación de la salud } & - Sedentarismo & $\mathrm{X}$ \\
\hline & Falta de motivación para el ejercicio físico & $\mathrm{X}$ \\
\hline & Desconocimiento de las cifras de tensión arterial & $\mathrm{X}$ \\
\hline & Desconocimiento de los fármacos antidepresivos & $\mathrm{X}$ \\
\hline
\end{tabular}

$\mathrm{AE}=$ Apoyo/Educación.

$\mathrm{PC}=$ Parcialmente compensatorio.

$\mathrm{TC}=$ Totalmente compensatorio. 
Cuadro II. Reconocimiento del autocuidado.

\section{Agencia de autocuidado}

Capacidades

- Capacidad de retención y asimilación de información

- Capacidad para desarrollar habilidades y aptitudes para la toma y registro de tensión arterial

- Responsabilidad para asumir el autocuidado

- Capacidad para discernir entre los diferentes grupos de alimentos

- Capacidad para elaborar un plan de autocuidado

- Capacidad para dar continuidad a su tratamiento farmacológico

- Capacidad para desarrollar actividad física

- Trasladarse para recibir atención médica cuando lo requiera

Déficit de autocuidado

Limitaciones

Falta de motivación y conocimiento sobre la depresión y sus complicaciones

- Desconocimiento sobre el régimen dietético

- Desconocimiento de los fármacos que conforman su tratamiento antidepresivo

- Falta de conocimiento sobre modificaciones en su estilo de vida
Cuadro III. Diagnósticos de enfermería.

Diagnóstico de enfermería Priorización

\section{Requisitos universales:}

Déficit alimenticio relacionado con la ingesta de dieta

hipercalórica y con gran cantidad de carbohidratos y grasas,

manifestado por no respetar la dieta establecida y sobrepeso

Déficit de actividad y reposo relacionados con el tipo de

actividad laboral desempeñada y enfermedades degenerativas

asociadas, manifestado por sedentarismo

Requisitos de desarrollo y desviación de la salud:

Desviación de la salud relacionada con deficiencia en el

apego al régimen terapéutico, manifestada por el desconocimiento de la misma y los signos de depresión

Nota: Los números indican el orden de importancia de los diagnósticos

Cuadro IV. Plan de atención de acuerdo con los diagnósticos de enfermería.

Requisito universal: Mantenimiento del aporte suficiente de alimento

Diagnóstico: Déficit alimenticio relacionado con la ingesta de dieta hipercalórica y con gran cantidad de grasa, manifestado por

no respetar la dieta establecida y sobrepeso

Objetivos:

Paciente

- Manifestar interés en el cumplimiento y conocimiento del régimen terapéutico

- Demostrar un aumento del nivel de conocimiento sobre el régimen dietético

Enfermería

- Orientar sobre los beneficios que proporciona el respetar el régimen dietético

Sistema:

- Apoyo/Educación

Actividades de autocuidado Intervenciones de enfermería

Evaluación

- Dialogar con la dietista para la elaboración de la dieta

- Aceptar la disminución de sal en la dieta

- Llevar a cabo un plan de control y registro de peso corpora

- Asistir a las entrevistas programadas

- Conocimiento básico sobre tratamiento

- Motivar y coordinar la elaboración de un plan para el control y registro del peso corpora

- Canalizar al paciente al Servicio de Dietología para la elaboración de su dieta

- Vigilar el cumplimiento del régimen dietético y peso corporal a través de entrevistas periódicas

- Programación de entrevistas para el

seguimiento y retroalimentación donde

se actualice periódicamente la información

Requisito universal: Mantenimiento suficiente para dormir y descansar

Diagnóstico: Déficit del sueño $\mathrm{r} / \mathrm{c}$ estado emocional $\mathrm{m} / \mathrm{p}$ ansiedad e insomnio

Objetivos:

Paciente

- Manifestar interés en el enfermo para evaluar la calidad del sueño, cumplimiento y conocimiento del régimen terapéutico

- Ayudar a mejorar el proceso de sueño para favorecer su descanso

Enfermería

- Orientar sobre los beneficios que proporciona el sueño

Sistema:

- Apoyo/Educación

Actividades de autocuidado

- Dialogar con el enfermo para la elaboración de un horario de sueño

- Disminución de distractores que impidan el sueño

- Llevar a cabo un plan de control de actividades antes del horario de la siesta

- Programar actividades deportivas, de lectura y terapias ocupacionales
Intervenciones de enfermería

- Motivar y coordinar la elaboración de un plan para el control del sueño

- Canalizar al paciente a actividades de terapia física y ocupacional

- Vigilar el cumplimiento del régimen de sueño y generar estrategias que lo favorezcan

- Programación de entrevistas para el seguimiento y retroalimentación donde se actualice periódicamente la información farmacológico

- Disposición a las intervenciones

- En las visitas periódicas, evaluar sobrepeso

- Conocimiento y registro de signos vitales
Evaluación

- Conocimiento básico sobre tratamiento farmacológico

- Disposición a las intervenciones

- En las visitas periódicas evaluar la técnica de toma de presión

- Conocimiento y registro de signos vitales 


\section{Fundamentación científica:}

a) La eliminación o reducción de distracciones ambientales favorecen la inducción del sueño, así como brindan todo el confort posible

b) En pacientes con dolor de espalda o miembros, el apoyo

en zonas de presión constante y cambio de posición mejora el descanso

c) Tomando en cuenta que no puede dormir por su estado emocional, se recomienda administrar ansiolíticos media hora antes para disminuir la imposibilidad de quedarse dormido o despertarse durante el descanso

Requisito universal: Mantenimiento del aporte para escoger ropa adecuada para vestirse y desvestirse

Diagnóstico: Déficit en el trastorno de la identidad $\mathrm{r} / \mathrm{c}$ estado emocional $\mathrm{m} / \mathrm{p}$ indiferencia en el autocuidado

Objetivos:

Paciente

- Manifestar interés en el enfermo para escoger la ropa adecuada para vestirse y desvestirse

- Ayudar a mejorar el proceso para escoger la ropa adecuada para vestirse y desvestirse

Enfermería

- Orientar sobre los beneficios que proporciona escoger la ropa adecuada para vestirse y desvestirse

Sistema:

- Apoyo/Educación

\begin{tabular}{|c|c|c|}
\hline Actividades de autocuidado & Intervenciones de enfermería & Evaluación \\
\hline $\begin{array}{l}\text { - Dialogar con el enfermo sobre la importancia } \\
\text { de escoger la ropa adecuada para vestirse } \\
\text { y desvestirse } \\
\text { - Disminución de la depresión que impida escoger } \\
\text { la ropa adecuada para vestirse y desvestirse } \\
\text { - Llevar a cabo un plan por semana para escoger } \\
\text { la ropa adecuada para vestirse y desvestirse } \\
\text { - Programar actividades sociales, deportivas y de } \\
\text { terapias ocupacionales que contribuyan a } \\
\text { escoger la ropa adecuada para vestirse y } \\
\text { desvestirse }\end{array}$ & $\begin{array}{l}\text { - Motivar al enfermo sobre su imagen y coordinar la } \\
\text { elaboración de un plan para escoger la ropa adecuada } \\
\text { para vestirse y desvestirse } \\
\text { - Canalizar al paciente a actividades de terapia física y } \\
\text { ocupacional } \\
\text { - Vigilar el cumplimiento del plan establecido y generar } \\
\text { estrategias que lo favorezcan } \\
\text { - Programación de entrevistas para el seguimiento y } \\
\text { retroalimentación donde se actualice periódicamente } \\
\text { la información } \\
\text { a) Realizar cambio de ropa hospitalaria y cama diario } \\
\text { b) Aplicación diaria de desodorante y cremas } \\
\text { c) Cuidados del cabello mediante el peinado } \\
\text { d) Manicura y pedicura } \\
\text { Fundamentación científica: La imagen corporal se refiere } \\
\text { a cómo una persona se siente con su aspecto físico. } \\
\text { La autoestima es la medida en que nos aceptamos y } \\
\text { valoramos nosotros mismos, esto puede influir sobre } \\
\text { nuestra forma de actuar. } \\
\text { El cambio de ropa es importante no sólo por la imagen } \\
\text { corporal que se desee expresar, también está relacionado } \\
\text { con la higiene personal: } \\
\text { a) El cambio de ropa disminuye el riesgo de infección por } \\
\text { falta de higiene y brinda confort } \\
\text { b) Los desodorantes evitan el mal olor por sudor y las cremas } \\
\text { fomentan el cuidado de la piel, la higiene y el confort } \\
\text { c) La higiene personal y cepillado del cabello evita molestias } \\
\text { nudos y fomentan el bienestar psicológico } \\
\text { d) El cuidado de uñas se debe realizar para brindar higiene y } \\
\text { confort previendo infecciones por hongos, principalmente }\end{array}$ & $\begin{array}{l}\text { - Conocimiento básico sobre tratamiento } \\
\text { farmacológico } \\
\text { - Disposición a las intervenciones } \\
\text { - Evaluar la sociabilización } \\
\text { por } \\
\text { y }\end{array}$ \\
\hline
\end{tabular}

Requisito universal: Mantenimiento del aporte para comunicarse con los demás para expresar emociones y temores

Diagnóstico: Déficit de la interacción social $\mathrm{r} / \mathrm{c}$ estado emocional $\mathrm{m} / \mathrm{p}$ el aislamiento

Objetivos:

Paciente

- Manifestar interés en el enfermo para comunicarse con los demás para expresar emociones y temores

- Ayudar a mejorar el proceso comunicarse con los demás para expresar emociones y temores

- Orientar sobre los beneficios que proporciona comunicarse con los demás para expresar emociones y temores

- Apoyo/Educación

Enfermería

- Orientar sobre los beneficios que proporciona comunicarse con los demás para expresar emociones y temores

Sistema:

- Apoyo/Educación

\begin{tabular}{|c|c|c|}
\hline Actividades de autocuidado & Intervenciones de enfermería & Evaluación \\
\hline $\begin{array}{l}\text { Dialogar con el enfermo sobre la importancia } \\
\text { de comunicarse con los demás para expresar } \\
\text { emociones y temores }\end{array}$ & $\begin{array}{l}\text { Motivar al enfermo sobre su imagen y coordinar la elaboración } \\
\text { de un plan para escoger la ropa adecuada para vestirse } \\
\text { y desvestirse }\end{array}$ & $\begin{array}{l}\text { - Conocimiento básico sobre tratamiento } \\
\text { farmacológico } \\
\text { - Disposición a las intervenciones }\end{array}$ \\
\hline
\end{tabular}


- Disminución de la depresión, comunicarse con los demás para expresar emociones y temores

- Llevar a cabo un plan para comunicarse con los demás para expresar emociones y temores

- Programar actividades sociales, deportivas y de terapias ocupacionales que contribuyan en la comunicación con los demás para expresar emociones y temores
- Canalizar al paciente a actividades de terapia física y ocupaciona

- Vigilar el cumplimiento del plan establecido y generar estrategias que lo favorezcan

- Programación de entrevistas para el seguimiento y retroalimentación donde se actualice periódicamente la información

a) Realizar cambio de ropa hospitalaria y cama diario

b) Aplicación diaria de desodorante y cremas

c) Cuidados del cabello mediante el peinado

d) Manicura y pedicura

Fundamentación científica: La imagen corporal se refiere a

cómo una persona se siente con su aspecto físico.

La autoestima es la medida en que nos aceptamos

valoramos nosotros mismos, esto puede influir sobre nuestra

forma de actuar.

El cambio de ropa es importante no sólo por la imagen

corporal que se desee expresar, también está relacionado

con la higiene personal:

a) El cambio de ropa disminuye el riesgo de infección por falta

de higiene y brinda confort

b) Los desodorantes evitan el mal olor por sudor y las cremas

ayudan al cuidado de la piel, fomentan la higiene y el confort

c) La higiene personal y cepillado del cabello evita molestias

por nudos y fomentan el bienestar psicológico

d) El cuidado de uñas se debe realizar para brindar higiene y

confort previendo de infecciones por hongos principalmente
Evaluar la sociabilización

Requisito universal: Mantenimiento del aporte emocional para vivir de acuerdo con los propios valores

Diagnóstico: Déficit emocional y sufrimiento espiritual r/c situación actual m/p desesperanza

Objetivos:

Paciente

- Manifestar interés en el enfermo y sus emociones para que viva de acuerdo con los propios valores

- Ayudar a mejorar el proceso emocional para reconocer y vivir de acuerdo con los propios valores

Enfermería

- Orientar sobre los beneficios que proporciona expresar las emociones para reconocer sus valores

Sistema:

- Apoyo/Educación

\begin{tabular}{|c|c|c|}
\hline Actividades de autocuidado & Intervenciones de enfermería & Evaluación \\
\hline $\begin{array}{l}\text { - Dialogar con el enfermo sobre la importancia } \\
\text { de expresar las emociones para reconocer } \\
\text { sus valores } \\
\text { - Programar actividades sociales, deportivas y } \\
\text { de terapias ocupacionales que contribuyan a } \\
\text { las emociones para vivir de acuerdo con los } \\
\text { propios valores }\end{array}$ & $\begin{array}{l}\text { - Motivar al enfermo a expresar sus emociones } \\
\text { - Canalizar al paciente a actividades de terapia física y } \\
\text { ocupacional } \\
\text { - Vigilar el cumplimiento del plan establecido y generar } \\
\text { estrategias que favorezcan sus emociones } \\
\text { - Ayudar al paciente a reencontrar una esperanza de vida } \\
\text { - Respetar las creencias del paciente. Permitir al paciente tener } \\
\text { imágenes simbólicas que lo reconforten durante su estancia } \\
\text { - Platicar con el paciente sobre sus creencias y religión } \\
\text { Fundamentación científica: Las creencias culturales en } \\
\text { los individuos son un complemento de apoyo emocional, ya } \\
\text { que la mayoría de las religiones o creencias se basan en } \\
\text { los principios de vida. El sufrimiento espiritual es una disrupción } \\
\text { en las primicias vitales del ser caracterizado por tristezas, } \\
\text { ansiedad, intranquilidad y desesperanza impidiendo el } \\
\text { bienestar integral } \\
\text { Fundamentación científica: } \\
\text { a) El respeto entre enfermera-paciente permite una buena } \\
\text { comunicación retroalimentativa } \\
\text { b) Las imágenes religiosas en algunos pacientes disminuyen } \\
\text { miedos y promueven un significado de continuar viviendo } \\
\text { c) La empatía relacionada con las creencias del paciente brinda } \\
\text { la oportunidad de disminuir el sufrimiento espiritual por medio } \\
\text { de la confianza }\end{array}$ & $\begin{array}{l}\text { - Reconocimiento básico sobre tratamiento } \\
\text { farmacológico y terapias ocupacionales } \\
\text { y evaluar la reintegración social } \\
\text { - Disposición a las intervenciones } \\
\text { - Evaluar la sociabilización }\end{array}$ \\
\hline
\end{tabular}

Los sistemas de enfermería servirán de guía para enfocar las intervenciones, y con ello, conocer los resultados de las mismas. Se deberá evaluar continuamente todo el proceso y sobre todo la implementación. La evaluación es la última etapa del plan de atención. El modelo de atención se debe basar en las cuatro etapas básicas señaladas anteriormente.

\section{CONCLUSIONES}

El modelo teórico de Orem contempla aspectos como: requisitos universales, requisitos de desarrollo y de desviación de la salud, que permiten valorar a la persona desde el aspecto físico, psicológico y social, obteniéndose 
diagnósticos de enfermería precisos para el enfermo con depresión para que, de esta manera, las intervenciones de enfermería sean aplicadas en todos los niveles de atención. En este caso, el nivel de prevención ha sido prioritario para que el paciente con depresión no se complique, o bien, se aborde adecuadamente en un segundo y tercer nivel de atención; esto es primordial, por lo que la enfermera debe adquirir la responsabilidad hacia esta población para que realice investigación y lleve a la práctica estrategias de atención continua. Se expuso de manera teórica un plan de atención al paciente con depresión y se sugiere su aplicación en la investigación con el propósito de validar con resultados estadísticos lo planteado teóricamente.

\section{BIBLIOGRAFÍA}

1. Jackson W. Historia de la melancolía y la depresión. Ed. Turner, Madrid, 1986: 56.

2. Moreno A, Medina ME. Tabaquismo y Depresión [PDF Internet]. Disponible en: http://www.inprf-cd.org.mx/pdf/sm3105/sm3105409.pdf

3. Mora ME, Borges G, Lara C, Benjet C, Blanco J, Fleiz C, Villatoro J, Rojas E, Zambrano J, Casanova L, Aguilar S. Prevalencia de trastornos mentales y uso de servicios: Resultados de la Encuesta Nacional de Epidemiología Psiquiátrica en México [Archivo PDF Internet]. Disponible en: http: //sap.salud.gob.mx/descargas/BIBLIOGRAFIA_COMPLETA/ Medina-Mora-PrevalenciaTrastornosMentales.pdf

4. Cruz A. Padece depresión 10\% de la población en México. La Jornada, 1 de junio 2008; Sociedad y justicia: 37.

5. Marriner A, Raile M. Modelos y teorías en enfermería. $6^{\text {a }}$ Edición. Editorial Elsevier, Madrid España; 2007: 267-295.

6. Lefevre RA. Aplicación del proceso enfermero. Editorial Masson, Barcelona; 2003. 